\title{
Preparation of cellulose fibres with antibacterial Ag-loading nano-SiO 2
}

\author{
WANG SHUHUA ${ }^{1,2}$, NIU RUNLIN ${ }^{1}$, JIA HUSHENG ${ }^{1,2}$, WEI LIQIAO ${ }^{1,2}$, DAIJINMING ${ }^{1,4}$, \\ LIU XUGUANG ${ }^{1,3}$ and XU BINGSHE ${ }^{1,2, *}$ \\ ${ }^{1}$ Key Laboratory of Interface Science and Engineering in Advanced Materials (Taiyuan University of Technology), \\ Ministry of Education, Taiyuan 030024, P.R China \\ ${ }^{2}$ College of Materials Science and Engineering, Taiyuan University of Technology, Taiyuan 030024, P.R. China \\ ${ }^{3}$ College of Chemistry and Chemical Engineering, Taiyuan University of Technology, Taiyuan 030024, P.R. China \\ ${ }^{4}$ College of Textile Engineering and Arts Taiyuan University of Technology, Yuci 030600, P.R. China
}

MS received 21 December 2009; revised 25 August 2010

\begin{abstract}
The antibacterial cellulose fibres with acrylamide polymerization and Ag-loading $\mathrm{SiO}_{2}$ nanoantibacterial materials were successfully prepared. The chemical structures and morphologies of antibacterial cellulose fibres were characterized by Fourier transformation infrared spectrum (FTIR), scanning electron microscopy (SEM), and transmission electron microscopy (TEM). The results showed that acrylamide was adsorbed on the surface of the cellulose fibres and formed a layer with thickness of 50-100 nm. The nano-SiO composite antibacterial materials were combined with cellulose fibres firmly by infiltrating into polyacrylamide layer about $100 \mathrm{~nm}$. The antibacterial cellulose fibres with antibacterial layer owned excellent antibacterial effect.
\end{abstract}

Keywords. Modification; cellulose fibres; Ag-loading $\mathrm{SiO}_{2}$; antibacterial property.

\section{Introduction}

Cellulose fibres are one of the excellent natural materials with wide application in composite materials (Seavey and Glasser 2001; Wang et al 2004; Pasquini et al 2008) and industrial materials. The surface properties of cellulose fibres are crucially important in many applications, the large amount of functional groups on the surface of cellulose fibres may be used for various activation processes. Hence, some attempts have been made to modify cellulose fibres for changing their surface property. For example, acrylic acid was grafted on the surface of cellulose fibres to improve their exchange or adsorption capacity (Liu et al 2002; Fadhel et al 2006). Silver ions have long been known to have strong inhibitory and bactericidal effects as well as a broad spectrum of antimicrobial activities (Kawashita et al 2000). Therefore, silver has been commercially used to take advantage of its antibacterial properties. Ag-loading nano- $\mathrm{SiO}_{2}$ as an antibacterial materials owned excellent antibacterial effects and were studied by many researchers (Jeon et al 2003; Oh et al 2006). In recent years antibacterial cellulose fibres have been a focus in the field of materials, but few reports can be found about the grafting of Ag-loading $\mathrm{SiO}_{2}$ nano-

\footnotetext{
*Author for correspondence (wsh73.student $@$ sina.com)
}

antibacterial materials on cellulose fibres surface. In this paper, the antibacterial cellulose fibres were prepared and the antibacterial property and structure were characterized.

\section{Experimental}

\subsection{Material}

The cellulose fibres were obtained from Taiyuan Pinde Company, Taiyuan. Nano-antibacterial materials used in the experiment were prepared by adsorption methodology in our laboratory (Wang et al 2007). Potassium permanganate $\left(\mathrm{KMnO}_{4}\right)$, acrylamide and sodium hydroxide were of analytical grade. Escherichia coli (E. coli) (ATCC 8739) and Staphylococcus aureus (S. aureus) (ATCC 6538) were provided by Shanxi Medical University, Taiyuan.

\subsection{Preparation of modified cellulose fibres by grafting acrylamide}

The modification of cellulose fibres was performed in three consecutive steps. In the first step $(15 \mathrm{~min})$, the fibres were pre-modified in $\mathrm{KMnO}_{4}$ solution at $50^{\circ} \mathrm{C}$, then washing until the water was colorless. In the second step, the pre-modified fibres were dipped in acrylamide solution at $70^{\circ} \mathrm{C}$ for $1.5 \mathrm{~h}$, which was adjusted with $\mathrm{H}_{2} \mathrm{SO}_{4}$ to give a $\mathrm{pH}$ value of $4-5$. Modified fibres were 
washed with the mixture of ethanol and water (ethanol: water $=1: 4 \mathrm{v} / \mathrm{v}$ ) to remove the unfixed polyacrylamide, then washed and dried.

In the experiments, the $\mathrm{KMnO}_{4}$ as the redox-initiator, the free radical were introduced onto the surface and grafted of acrylamide onto cellulose fibres.

The main reactions are shown as follows.

Reaction 1 (cellulose fibres pre-oxidation):

$$
\mathrm{Cell}+\mathrm{KMn}_{4} \rightarrow \mathrm{Cell} \bullet+\mathrm{Mn}^{2+} .
$$

Reaction 2 (graft-polymerization of acrylamide):

$$
\begin{aligned}
& \text { Cell } \bullet+\mathrm{NH}_{2}-\prod_{\mathrm{C}}^{\mathrm{O}}-\mathrm{CH}=\mathrm{CH}_{2}
\end{aligned}
$$

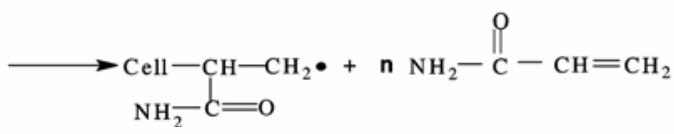

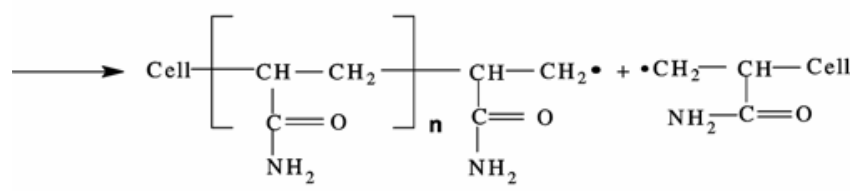

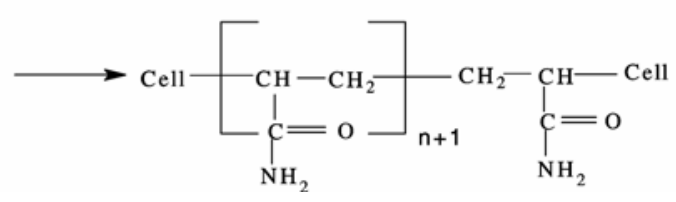

\subsection{Preparation of antibacterial cellulose fibres}

Antibacterial functional suspension was prepared by adding $5 \mathrm{~g}$ of as-prepared $\mathrm{Ag}$-loaded nano- $\mathrm{SiO}_{2}$ and $5 \mathrm{ml}$ of $\mathrm{KH}-550\left(\mathrm{H}_{2} \mathrm{~N}\left(\mathrm{CH}_{2}\right)_{3} \mathrm{Si}\left(\mathrm{OC}_{2} \mathrm{H}_{5}\right)_{3}\right)$ into $200 \mathrm{ml}$ of distilled water with $\mathrm{pH}$ value adjusted to 6 , then keeping stirred at $60^{\circ} \mathrm{C}$ for $15 \mathrm{~min}$. The modified cellulose fibres were impregnated in the antibacterial functional suspension for $1 \mathrm{~h}$, and then washed with distilled water for 20 times and dried at $80^{\circ} \mathrm{C}$ for $4 \mathrm{~h}$. The overall scheme of grafting the nano- $\mathrm{SiO}_{2}$ composite antibacterial materials on the cellulose fibres surface is shown in figure 1. There are two main steps in the grafting reaction process. The first step is the antibacterial materials are adsorbed to cellulose fibres surface by hydrogen bond. In the second step, the stable chemical bond is formed by reaction between an amino group of modified cellulose fibres and a hydroxyl group of antibacterial materials with elimination of water.

\subsection{Measurements}

For SEM analysis, the fibres samples were sputtered with gold and then characterized by a scanning electron microscope (JEM-6700F; JEOL, Japan), accelerating voltage at $15 \mathrm{kV}$.
The microstructures of the samples were characterized using a high-resolution transmission electron microscope (HRTEM-2010, JEOL, Japan).

The IR analysis was recorded Fourier transformed infrared spectrum (FT-IR1730, PE, USA).

The antibacterial properties of antibacterial cellulose fibres were measured by shake flask testing. E. coli (ATCC8099) and S. aureus (ATCC6538) were selected as indicators of experimental bacteria. The antibacterial performance of the antibacterial cellulose fibres was measured by shake flask testing method (Seavey et al 2001). The antibacterial effect was calculated using the following relationship

$$
R \%=(\mathrm{A}-\mathrm{B}) / \mathrm{A} \times 100 \% .
$$

where $R$ is antibacterial effect (\%), A and B are the mean numbers of bacteria in $0.5 \mathrm{ml}$ of the mixed solutions before and after shaking.

The reported data were the average value of three parallel runs.

\section{Results and discussion}

\subsection{Surface morphologies of antibacterial cellulose fibres}

SEM analysis was conducted to characterize possible changes on the surface of the cellulose fibres. Figure 2 Shows the SEM images of parent, pre-modified, acylamide graft-polymerized and antibacterial cellulose fibres. It can be seen that there were clear-cut groove on the surface of parent cellulose fibres. After pre-modified, the cellulose fibres had no obvious change in surface morphology (figure 2b). However, the surface of modified cellulose fibres was covered by polyacrylamide (figure $2 \mathrm{c}$ ). Figure $2 \mathrm{~d}$ is the SEM image of the antibacterial cellulose fibres grafted the nano- $\mathrm{SiO}_{2}$ composite antibacterial materials. As predicted, nano- $\mathrm{SiO}_{2}$ composite antibacterial particles were well dispersed on the surface of cellulose fibres and a compact antibacterial layer be formed on the cellulose fibres surface. A few antibacterial particles became agglomerated because of their high surface free energy, so bigger agglomerates were observed on cellulose fibres surface.

\subsection{The microstructure characterization of antibacterial cellulose fibres}

Figure 3 is the TEM images of the cross-section of individual cellulose fibre before and after graft-polymerization. It shows that the layer of polymer was formed with a depth of about from $50 \mathrm{~nm}$ to $110 \mathrm{~nm}$, as shown in figure $3 \mathrm{~b}$. Region 2 is the layer of polyacrylamide, region 1 is the cortical layer of cellulose fibres. 


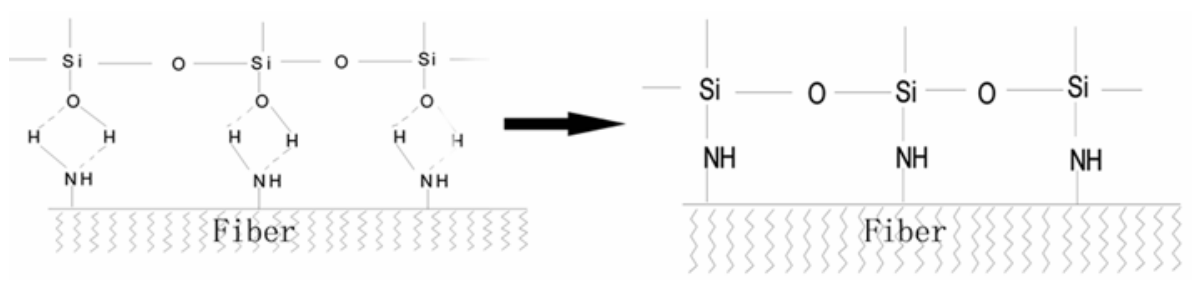

Figure 1. Schematic illustration of cellulose fibres surface grafting with nano- $\mathrm{SiO}_{2}$ composite antibacterial materials.

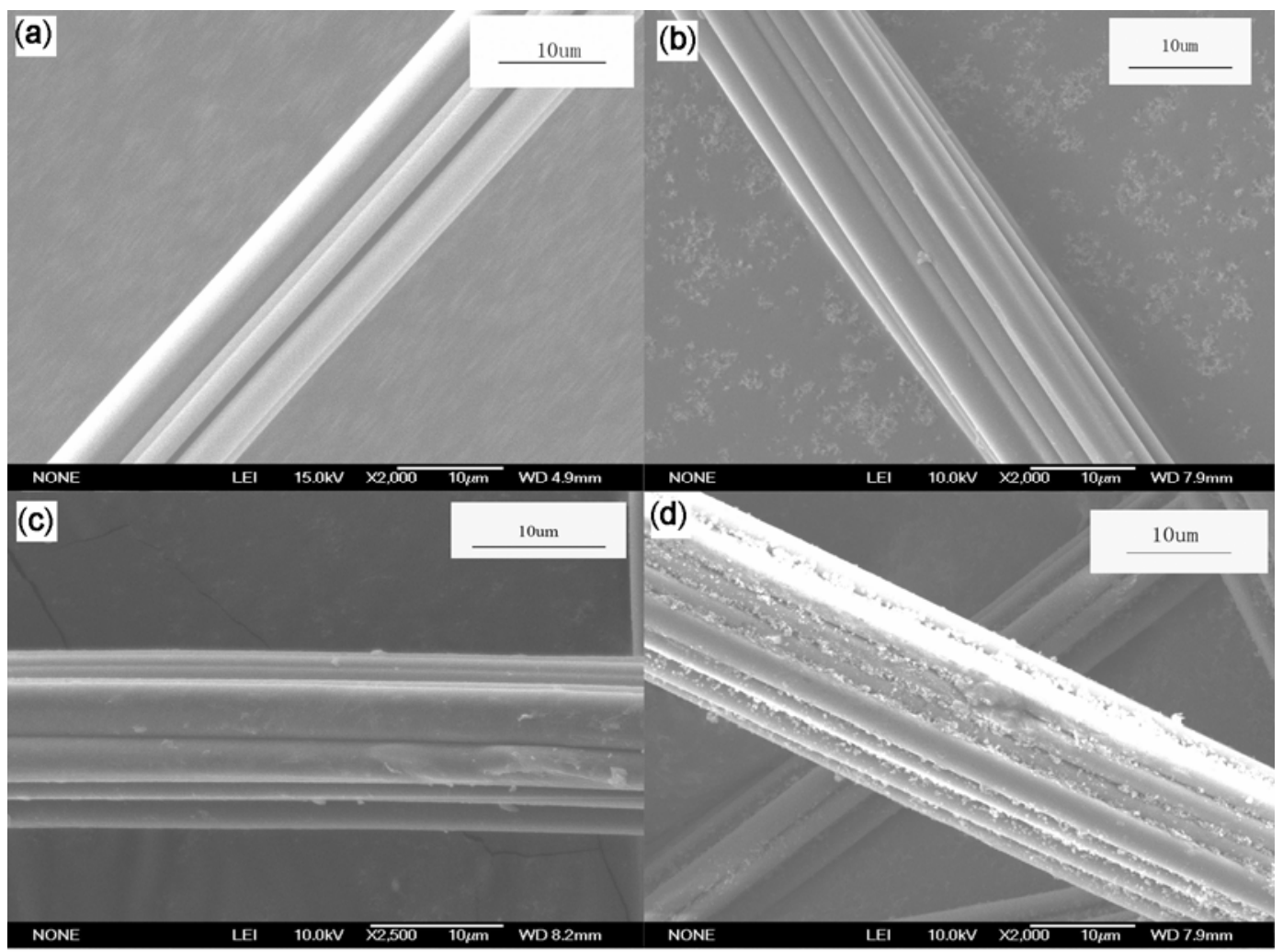

Figure 2. SEM images of cellulose fibres: (a) parent; (b) pre-modified; (c) polymerization grafted and (d) grafted by Ag-loading $\mathrm{SiO}_{2}$ nano-antibacterial materials.

Figures $3 \mathrm{c}$ and $\mathrm{d}$ is the TEM images of the crosssection of individual antibacterial cellulose fiber, in which a new layer of antibacterial materials was clearly observed. Region 1 is the cortical layer of cellulose fibres. Region 2 is the antibacterial layer of cellulose fibres, the black materials in (d) are the grafted antibacterial functional layer with thickness of about $200 \mathrm{~nm}$. The white part is the cortical layer of cellulose fibres. It can be seen that nano-antibacterial materials infiltrated into polyacrylamide layer in depth no less than $100 \mathrm{~nm}$ and integrated with cellulose fibres firmly.

\subsection{Chemical structures of antibacterial cellulose fibres}

FTIR spectra of the samples were obtained to investigate the molecular structure changes of cellulose fibres. The spectra of the parent, pre-modified, acrylamide-grafted and $\mathrm{SiO}_{2}$-grafted cellulose fibres in $4000-2000 \mathrm{~cm}^{-1}$ and $2000-400 \mathrm{~cm}^{-1}$ are shown in figure 4 , respectively.

In figure $4 \mathrm{~A}$, the bands at $3450 \mathrm{~cm}^{-1}$ and $2920 \mathrm{~cm}^{-1}$ show the presence of $\mathrm{C}-\mathrm{H}$ and $-\mathrm{OH}$ in parent cellulose fibres, no significant changes can be observed for premodified sample. However, the spectra of grafted cellulose fibres (curve c) show broader band at $3450 \mathrm{~cm}^{-1}$ and a shoulder band at $3200 \mathrm{~cm}^{-1}$, which is attributed to $-\mathrm{NH}$ vibration. Compared with curves a and $b$, two strong bands in curves $\mathrm{c}$ and $\mathrm{d}$ can be observed at $2370 \mathrm{~cm}^{-1}$ and $2332 \mathrm{~cm}^{-1}$ (secondary amine), it proves that acrylamide showed a certain polymerization degree after graftpolymerization.

In figure $4 \mathrm{~B}$, compared with parent cellulose fibres (curve a), pre-modified cellulose fibres (curve b) had no 


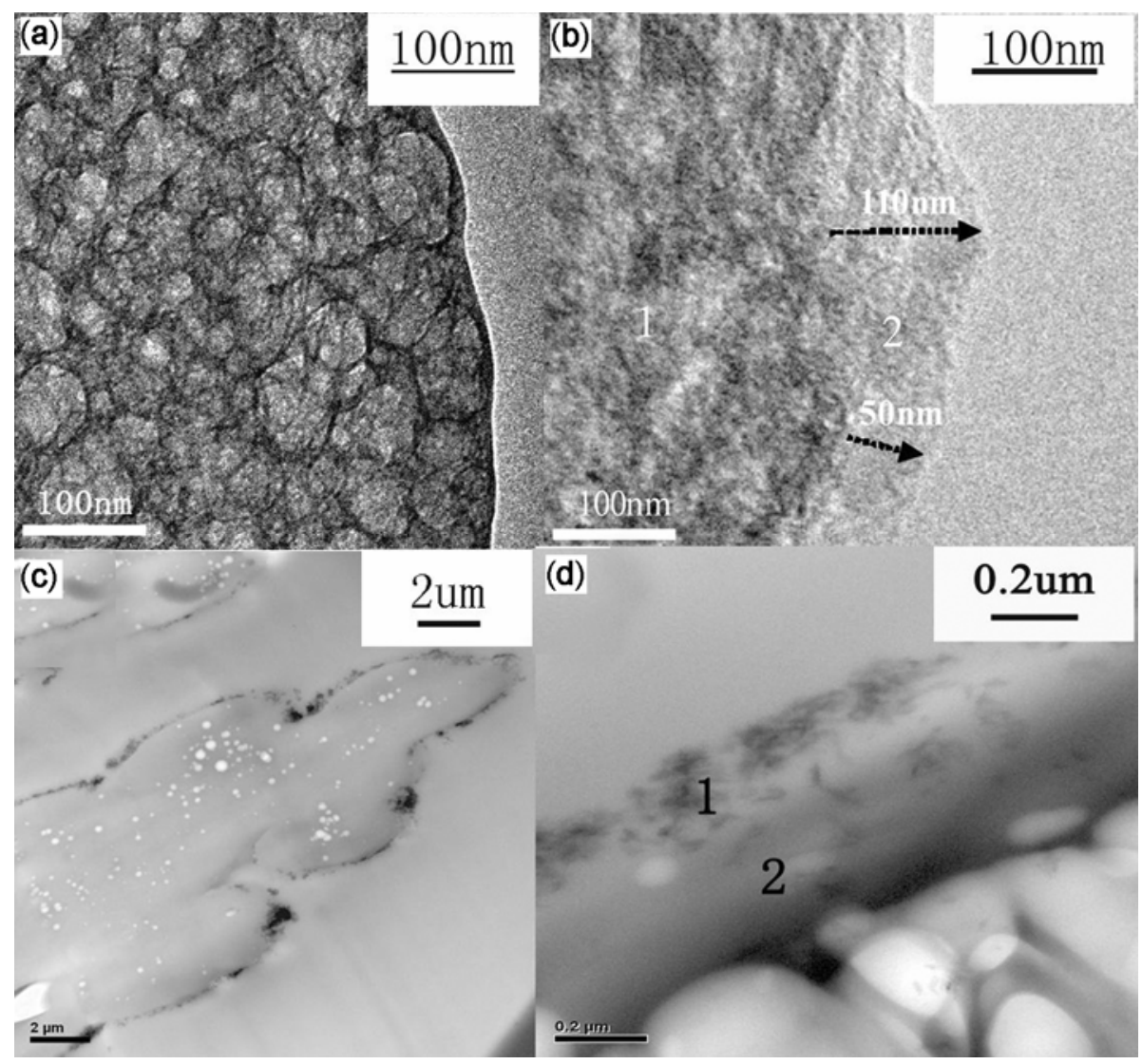

Figure 3. TEM images of the cross-section of cellulose fibres: (a) parent; (b) polymerization modified grafted; (c) grafted by Ag-loading $\mathrm{SiO}_{2}$ nano-antibacterial materials; (d) grafted by Ag-loading $\mathrm{SiO}_{2}$ nano-antibacterial materials at higher magnification.)

obvious changes. In the curve of acrylamide-grafted fibres (curve c), the main changes (indicated by arrowhead) are the increase in the band intensity at 1647 and $670 \mathrm{~cm}^{-1}$ (attributed to $-\mathrm{NH}$ ). Because of acrylamide polymerization on the surface of cellulose fibres, the band at $1645 \mathrm{~cm}^{-1}$ split into two bands at $1647 \mathrm{~cm}^{-1}$ (-OH bending of bound water) and $1684 \mathrm{~cm}^{-1}$ (the carbonyl in acrylamide), meanwhile, some new bands can occur at $1560,1540,1516 \mathrm{~cm}^{-1}$ (-NH).

Compared with parent cellulose fibres, the antibacterial cellulose fibres were quite different in the IR spectra (figure $4 \mathrm{~B}$, curve d). The absorption peaks of $\mathrm{SiO}_{2}$ appeared at $670 \mathrm{~cm}^{-1}$ and $471 \mathrm{~cm}^{-1}$. A new characteristic band appeared at $1157 \mathrm{~cm}^{-1}$, belonging to $\mathrm{Si}-\mathrm{O}-\mathrm{Si}$ strentching vibration band (Wang et al 2007). Therefore, it can be suggested that the partial antibacterial materials were adsorbed to cellulose fibres.

\subsection{The antibacterial properties of antibacterial cellulose fibres}

The modification of acrylamide gave the cellulose fibres certain antibacterial efficiency, that is, against $E$. coli and
$S$. aureus is $21 \%$ and $26 \%$, respectively. According to the State Standard GB1598-1995 for the evaluation of disinfections and antibacterial properties defined by the Ministry of Health, China, if the difference of the antibacterial ratios between the tested specimen and the referred one is over $26 \%$, the tested specimen can be considered to be of antibacterial activity. Hence modified cellulose fibres by grafting acrylamide have not been used as antibacterial fibres.

The antibacterial effect was greatly raised when the modification cellulose fibres were treated by Ag-loaded nano- $\mathrm{SiO}_{2}$. Silver content of Ag-loading $\mathrm{SiO}_{2}$ nanoantibacterial agent was $0 \cdot 8 \%$ through atomic absorption spectrum (ICPS) (LB-8410) whose antibacterial effect was $99 \%$. So we calculated the Ag content loaded on the antibacterial cellulose fibres. The effect of the silver concentration of antibacterial agent on the antibacterial activity of wool fibres is shown in table 1 . It can be seen that the antibacterial ratio increased with increasing silver content of antibacterial cellulose fibres. When the silver content was $0.034 \%$, the antibacterial ratio was up to $98 \%$ against $E$. coli and $97 \%$ against $S$. aureus. However, when the concentration was over $0.34 \mathrm{~g} / \mathrm{ml}$, the antibacterial ratio only showed a slow increase or no increase. 

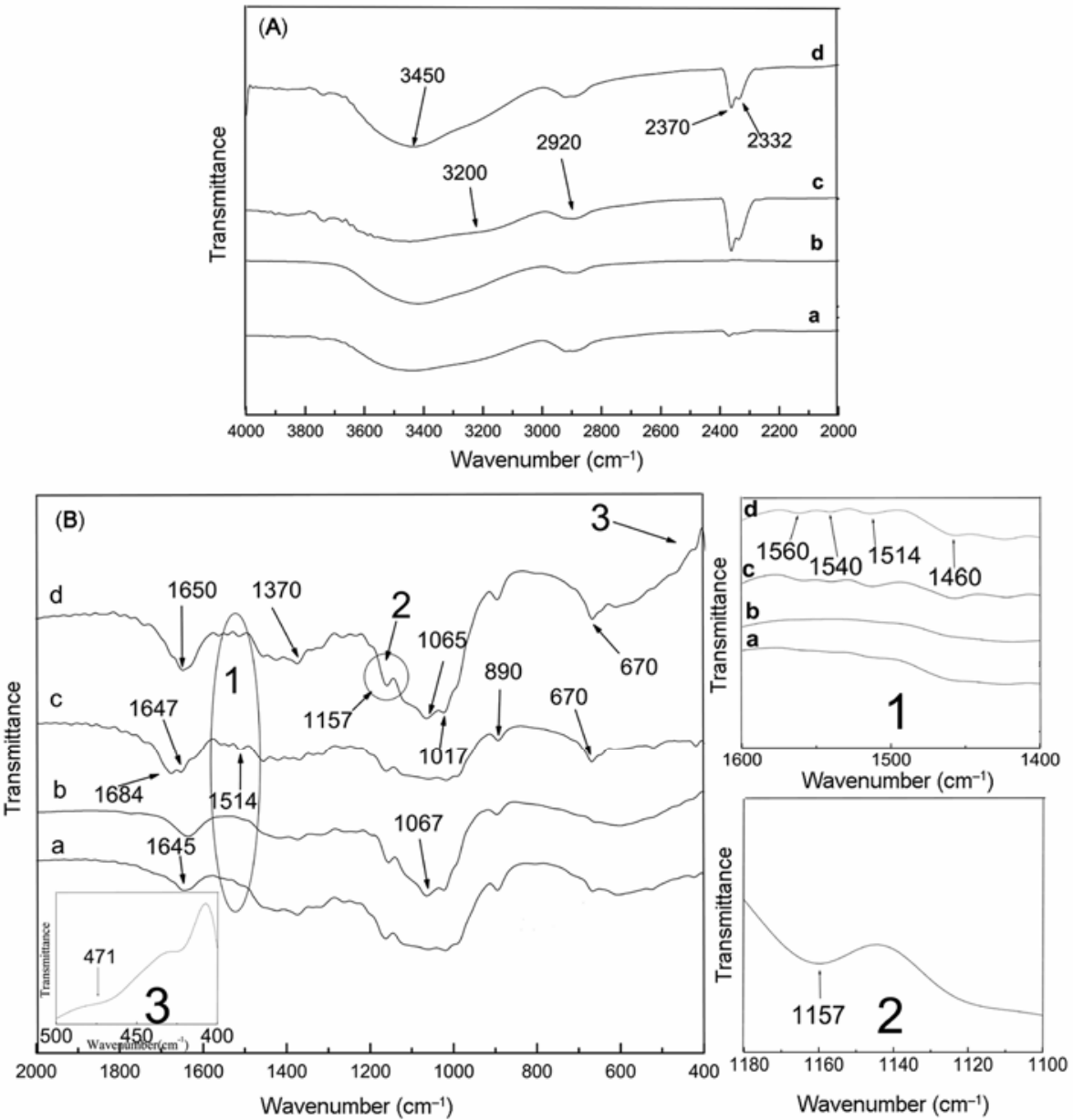

Figure 4. (A) FTIR spectra of parent (a), pre-modified (b), graft-polymerized (c) and $\mathrm{SiO}_{2}$ grafted (d) cellulose fibres: (A) 4000-2000 $\mathrm{cm}^{-1}$ FTIR spectra, (B) 2000-400 $\mathrm{cm}^{-1}$ FTIR spectra.

Table 1. The antibacterial ratios of antibacterial cellulose with different silver content.

\begin{tabular}{|c|c|c|c|c|}
\hline \multirow[b]{3}{*}{ Silver content $(\%)$} & \multicolumn{4}{|c|}{ Antibacterial ratio $(\%)^{*}$} \\
\hline & \multicolumn{2}{|c|}{ E. coli } & \multicolumn{2}{|c|}{ S. aureus } \\
\hline & Mean value & Standard deviation & Mean value & Standard deviation \\
\hline $0 \cdot 0$ & 0 & - & 0 & - \\
\hline $0 \cdot 011$ & 54 & $2 \cdot 3$ & 41 & $2 \cdot 3$ \\
\hline $0 \cdot 019$ & 74 & $2 \cdot 5$ & 74 & $3 \cdot 6$ \\
\hline $0 \cdot 024$ & 80 & $2 \cdot 4$ & 76 & $2 \cdot 5$ \\
\hline 0.034 & 98 & $2 \cdot 2$ & 97 & $2 \cdot 4$ \\
\hline $0 \cdot 036$ & 98 & $2 \cdot 3$ & 98 & $2 \cdot 2$ \\
\hline
\end{tabular}

Above results suggest that an adequate antibacterial agent on wool surface is necessary for the inhibition of the bacterial growth.

\section{Conclusions}

Cellulose fibres were modified by grafting acrylamide polymerization. Modified cellulose fibres showed a significant change in chemical structure and physical mor- phology. The nano- $\mathrm{SiO}_{2}$ composite antibacterial materials was grafted and well dispersed on the surface of the cellulose fibres. The nano- $\mathrm{SiO}_{2}$ composite antibacterial materials formed an antibacterial layer with thickness of $200 \mathrm{~nm}$ on the antibacterial cellulose fibres surface and infiltrated into acrylamide layer in depth no less than $100 \mathrm{~nm}$ and integrated with cellulose fibres firmly. The antibacterial cellulose fibres showed excellent antibacterial activity against the tested germs. 


\section{Acknowledgements}

This work was supported by the International cooperation Program of Shanxi province (No. 2007081029 and 2009081046) and Key Laboratory of Materials Interface Science and Engineering, Ministry of Education, Taiyuan University of Technology, Taiyuan Shanxi, China.

\section{References}

Fadhel A, Sami B and Jalel L 2006 Purif. Technol. 52332 Jeon H J, Yi S C and Oh S G 2003 Biomaterials 244921
Kawashita M, Tsuneyama S, Miyaji F, Kokubo T, Kozuka H and Yamamoto K 2000 Biomaterials 21393

Liu X H, Huang C R and Wei Y 2002 China Synthetic Fibre Industry 2529

Oh S D, Lee S, Choi S H and Lee I S 2006 Colloids \& Surf. A275 228

Pasquini D, Teixeira E M, Curveloa A A S, Belgacem M N and Dufresne A 2008 Compos. Sci. Technol. 68193

Seavey K C and Glasser W G 2001 Cell 8161

Wang S H, Hou W S and Xu B S 2007 Surf. \& Coatings Technol. 202460

Wang Z X, Shen Q and Gu QF 2004 Carbohyd. Polym. 57 415 